\title{
LUT
}

Lappeenranta

University of Technology

\section{Technological Landscape and Ideation in the Field of Waste Separation with Help of TRIZ}

\author{
Ivanovskis Luka, Kärki Timo, Lahtela Ville
}

This is a Final draft

version of a publication

published by Springer, Cham

in New Opportunities for Innovation Breakthroughs for Developing Countries and Emerging Economies. TFC 2019. IFIP Advances in Information and Communication Technology

DOI: 10.1007/978-3-030-32497-1_27

Copyright of the original publication: (c) 2019 Springer Nature Switzerland AG.

Please cite the publication as follows:

Ivanovskis L., Kärki T., Lahtela V. (2019) Technological Landscape and Ideation in the Field of Waste Separation with Help of TRIZ. In: Benmoussa R., De Guio R., Dubois S., Koziołek S. (eds) New Opportunities for Innovation Breakthroughs for Developing Countries and Emerging Economies. TFC 2019. IFIP Advances in Information and Communication Technology, vol 572. Sprinaer. Cham. DOI: 10.1007/978-3-030-32497-1 27 
Final draft of manuscript

\title{
Technological Landscape and Ideation in the Field of Waste Separation with Help of TRIZ
}

Luka Ivanovskis, Timo Kärki, Ville Lahtela

Fiber Composite Laboratory, LUT University, P.O. Box 20, FI-53851 Lappeenranta, Finland

\section{Keywords:}

Effects database

Function-oriented search

Material recovery

Waste separation

\begin{abstract}
Solid waste separation is an imperative part for closing material flow under principles of the circular economy. A multitude of technologies are at a different stage of development to perform this task and meet performance requirements at extracting valuable resources from waste streams. A thorough review of available options and possibilities for future development was required to pursue further progress. TRIZ (from Russian abbreviation: Theory of Inventive Problem Solving) methodology facilitated drawing a technological landscape for state of the art in solid waste separation for material recovery, as well as generating new directions of research. From the vast collection of TRIZ tools for systematic invention two approaches helped in the survey: effects databases and function-oriented search. Positive outcomes of their application include systematization of available knowledge, identification of potentially useful phenomena and getting ideas for the adoption of technologies from other domains. The case illustrates how rather loose (due to the broadness of the topic) application of TRIZ methods results in fruitful insights. A view at the effects databases as a collection of technological "genes" is given.
\end{abstract}

\section{Introduction}

Concerns about scarce natural resources and ever-increasing amount of waste promote the implementation of the circular economy principle. One of the key points of the circular economy is material recovery from waste for further reuse in new products. Material liberation and separation are the essential prerequisites for closing the loop of material flow, as materials recycled from waste should attain a certain level of purity to be used for new products of same quality [1]. Separation of materials from commingled waste streams poses technological challenges and addressing them requires an indepth knowledge of state of the art in both industry and academia. Further development of technical means for waste separation cannot progress without novel ideas. Acquiring such knowledge together with insights into the future prospective is the task a research institution faces as it enters the field and seeks for its own path.

Various decision support tools were developed in the past for the recycling field. They suggested optimal waste treatment scenario [2], predicted achievable material recovery from complex products [3], maximized profit by rearranging waste separators in sequence [4]. Recent reviews of separation technologies discussed their pros and cons in a narrative manner $[1,5,6]$, attempting to address one of the significant issues with technological road mapping - capturing large sets of quantitative and qualitative data [7]. An idea generation tool for the treatment of a particular waste through structured web search queries of related terms was realized in [8]. Methods of technological road mapping for manufacturing enterprises fall into four categories according to [9]:

- $\quad$ Causal models,

- Phenomenological models, 
- Intuitive models,

- Monitoring and mapping.

When technological forecasting is taken more broadly (including research interests, for example), the analysis itself can be classified in nine dimensions (driver, locus etc.), whereas the methods fall into more than ten families of distinct purposes [10].

Review and comparison of technologies for mechanical separation of solid waste was done to determine their capabilities and limitations. The broadness of the topic and technological diversity required auxiliary tools to make the analysis systematic and facilitate the generation of feasible ideas. Tools applied in the study belong to the methods of modern TRIZ. TRIZ (from Russian abbreviation: Theory of Inventive Problem Solving) methodology provides a set of tools for systematic invention given the defined problems and goals [11]. Wide research question and limited experience in TRIZ narrowed the arsenal used in the study down to a couple of tools: databases of physical effects and the FunctionOriented Search (FOS) [12]. Presenting the outcomes of their fragmentary application is the subject of the present paper.

\section{Methods}

The study [13] consisted of two parts: a comparative review of technologies based on literature search and suggestion of new principles that could potentially improve waste separation. Although they have a different meaning, the latter stems from the former one, as it will become clear from the following description.

The use of effects databases had several goals in mind:

1. For every technology, there was found a set of effects that play the role in its operation. This could help to categorize technologies and draw boundaries between technologies of a similar kind.

2. Representation of technologies as the sets of effects allowed to see better the evolution path among technology families, especially for old separation technologies stemming from the centuries-long mining industry.

3. A list of effects (together with technologies) would suggest those that remain unused in modern recycling, some of which could find a real application.

As it can be seen already, the same database can have a positive effect on both review and idea generation parts of the study. The list of effects and technologies was taken from the online databases by Aulive [14] and Oxford Creativity [15]. Altogether they made about 600 entries (with repetitions). The idea of the effect lists was extended with a list of material properties that focused on the intrinsic properties by which materials were distinguished from each other. The list of material properties was taken from filter values of the material search database Matmatch [16].

For objective comparison of technology the diverse information found was stored in .json format text file in a nested form (see Figure 1), namely a record of every technology constituted a tree, with the technology basic definition and associated properties (like target material properties, effects, affecting factors, maturity level) in the root and splitting into branches with instances of research or industrial devices and associated performance figures, both categorical (like materials sorted) and numerical (purity grade, recovery rate etc.). A custom piece of software was written in Julia language to parse the file and extract information about technologies meeting the search criteria in both tabular and graphical form (see 
example in Figure 2. Information from 199 scientific publications, patents and commercial leaflets about more than 40 technologies was collected in the database.

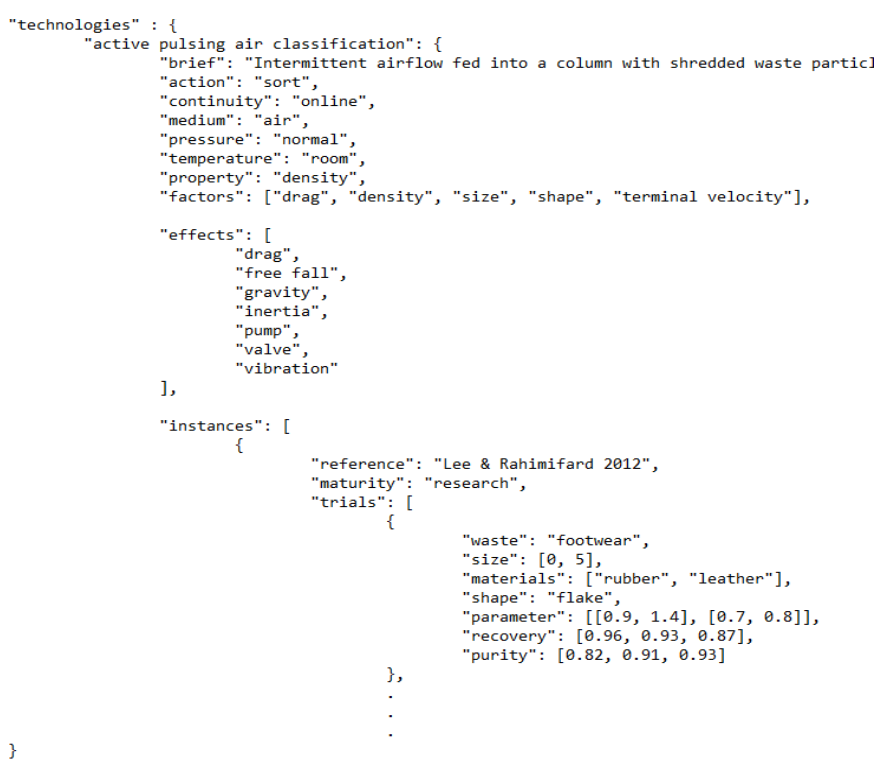

Figure 1. Excerpt from the .json text file, showing the nested structure of the database.

Finally, the FOS was used to recruit working principles and technologies used elsewhere outside recycling. A couple of striking similarities between waste sorting and other fields of technology will be shown.

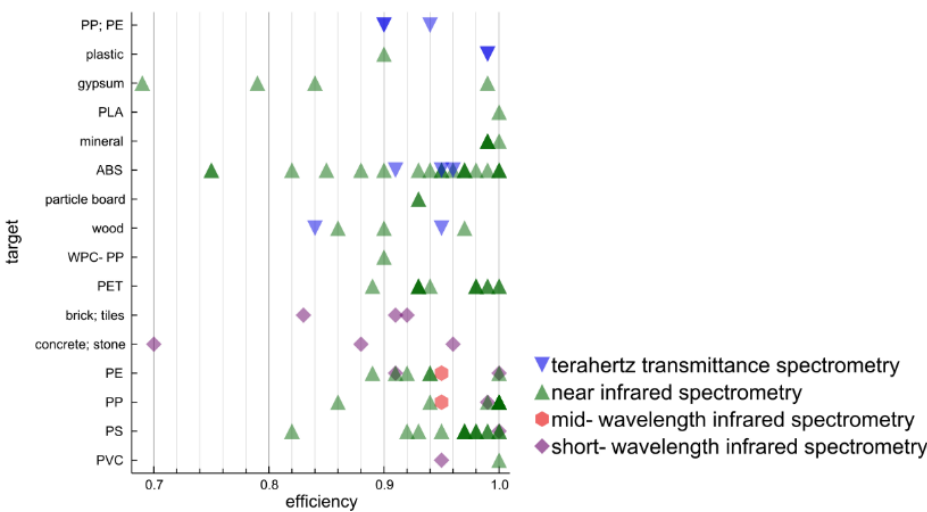

Figure 2. The efficiency of several optical spectrometry methods for classifying different materials, plot generated straight from the database.

\section{Useful outcomes of TRIZ}

In the following, some examples of the applied TRIZ procedures are given alongside with observations on their applicability. 


\subsection{Effects for systematics}

The organizing power of matching technologies with effects can be illustrated with the family of separation methods based on the difference in density. The very basic sink-float process lets waste fragments to segregate depending on their buoyancy in a liquid. The process is rather slow and obscured by material wettability and fluid viscosity. The gravity force can be replaced with centrifugal force produced either in hydrocyclone [17] or decanter centrifuge [18]. Another approach to diminish the unwanted secondary factors is replacement of hydrostatic (Archimedes') force with hydrodynamic forces acting intermittently in the wet shaking table [19], multidune [20], water jigging [21] or wet fluidized bed processes [22]. It can be further subdivided whether the alternating acceleration is excited with vibrations or zigzag flow of fluid. Practically for every aforementioned wet process, a complementary dry separation process can be found. Again, the underlying effects make the match rather explicit, like the use of zigzag flow pattern in multidune and zigzag air classifier [23].

Recently some new density-based methods emerged that introduced considerable adaptivity to the ordinary sink-float methods. The principle that attained the greatest success is manipulation of the Archimedes' force by placing the separation medium in the magnetic field. Options for the fluid are a solution of paramagnetic salt [24] or ferrofluid - the suspension of iron particles in water [25]. The interaction between the magnetic fluid and the field alters the apparent density of the fluid so that its layers attain different density. This enables separation of materials into multiple fractions in one separation volume (material particles float at the different depth), as well as adjustment of the density ranges without altering the fluid itself. One step further from that is the creation of a virtual inclined plane in the tank by inclining the external magnetic field. In this case, no propulsion for the floating items is needed, since they slide downhill along the layer of constant fluid density. This shows the broadness the effects can be treated with to build analogies between the processes. Also, the macroscale of material separation in waste processing allows utilization of effects not applicable for solid handling in conventional process engineering (compare with [26]): Magnus effect (eddy current separation [27]), stick-slip effect (air tabling [28]), triboelectricity (triboelectrostatic separation [28]).

Certain difficulties were faced in the description of sensor-based sorting, as the underlying interaction between matter and electromagnetic (EM) radiation is not so well covered by the list of effects and a significant amount of knowhow is hidden behind the classification algorithms. Still, some systematization was possible as to which part of the EM spectrum is used, type of interaction, e.g. reflection, transmission or fluorescence etc.

\subsection{Unused effects and material properties}

A large part of the effects remain unused in recycling for a good reason - they have limited applicability, for example, they concern a very narrow class of materials under special conditions (like piezoelectric or electrets). Consequently, there is not much to say about those effects that did not fall into approximately 100 that were used or tested in recycling. The same holds for material properties that are often challenging for continuous measurement (take stiffness, for example). However, a few effects and material properties have drawn attention and provided ideas for their application.

The first one is the Coandă effect - adherence of a fluid jet to a convex surface it flows along. An illustrative example of its use is the enhancement of lifting force of an airplane by deflecting the stream exhausted from the engines by utilizing its attachment to the upper surface of flaps pointed downwards (when being in the takeoff or landing position). At this point, it becomes apparent that deflection of stream produces centrifugal action on the particles following the very same stream. There are commercial devices based on this principle, such as Coandă screen [29] and Coandă classifier [30], see Figure 3. Coandă 
screen is a self-cleaning filter used to remove sediment from water stream without clogging and at high throughput. This device does not seem to be used in recycling, although it can help to recirculate the separation medium in the wet density-based separation methods without compromising their performance. Coandă (or elbow jet) classifier is laboratory equipment for separation of powders into fine, medium and coarse fractions by blowing them with the air around a rounded corner. Only the finest (and lightest) particles are able to follow the corner, while the heavier ones exit the stream and end up in other sectors. The size of powders classified $(<200 \mu \mathrm{m})$ is below that typical for waste, but targeted development may result in separators for finely granulated printed circuited boards (PCB), for example.

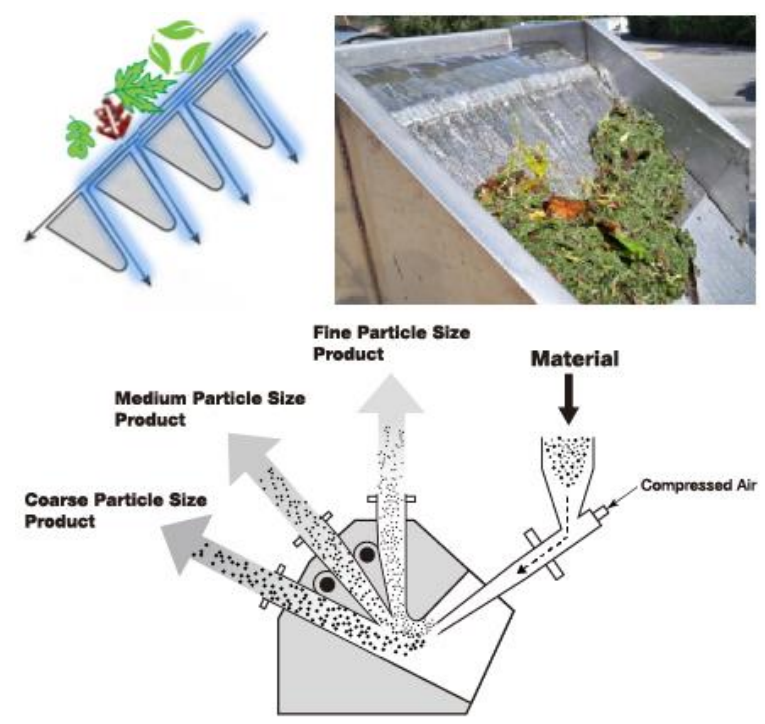

Figure 3. Coandă effect applications: Coandă screen (above) [29], Coandă classifier (below) [30].

A rather unexpected benefit of going through the effects list was finding separation technologies that did not appear in a targeted search. For example, supercritical fluids possess several properties highly desired for density separation medium, namely: liquid-like density, gas-like viscosity and compressibility, thus making adjustment of density rather straightforward. Indeed, the search about supercritical fluids revealed one technology that was developed and patented back in the 1990s for separation of plastics by density [31]. The technology has not gained popularity, but without it, the technological landscape would be incomplete. Paramagnetism is yet another term that deserves attention as it leads to using gas for density-based separation. Oxygen possesses paramagnetic properties, which makes it possible to suspend solids in oxygen or even air in a high-gradient magnetic field with slight pressurization (tens of bars). The need for strong magnetic fields can be further reduced when using liquid oxygen-nitrogen mixture (which is essentially the product of air liquefaction) as separation medium [32]. The idea may benefit from the synergy with the other features of cryogenics:

- $\quad$ cryocomminution of waste allows to crush and liberate materials from certain kinds of waste more effectively [33];

- $\quad$ high-temperature superconducting magnets outperform permanent magnets and they work at the temperature of liquid nitrogen [34];

- $\quad$ liquid nitrogen enables dry, eco-friendly cleaning (already used in industry [35]), so it can be applied to the waste as well. 
As cryogenic technology and superconductors are gaining widespread use, they may finally enter the field of waste processing.

It is quite challenging to find a new material property that is both sufficient for material discrimination and has adequate means for measurement. Still, two ideas got support for their feasibility. The first one should distinguish between thermoplastics with low and high softening temperatures. Among other test procedures, Vicat hardness test is used for plastics. Vicat softening temperature measures the point when the plastic softens enough to be penetrated by the steel test probe [36]. This test procedure could be approximated for continuous operation on a conveyor, analogue to the way the PaperSpike [37] mechanism separates cardboard items from paper, see Figure 4.
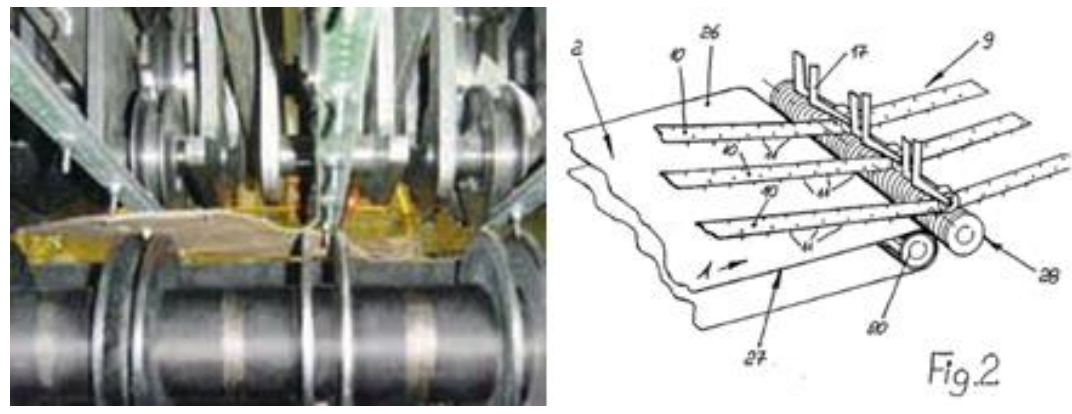

Figure 4. PaperSpike pierce a piece of cardboard (left) [37], view of mechanism (right) [38].

Another material property that becomes attractive for discrimination between materials is the refractive index. Advances in the theory of light reflection resulted in computational algorithms for estimation of refractive index from several photos of an object [39]. It is worth mentioning that one of the key drivers for research here is photorealistic image rendering (for computer games as well). Given that the refractive index was successfully measured by this technique for objects like tomato and apple [40], it might help in sensor-based classification of materials in waste.

\subsection{FOS: security scanners and printers in recycling}

Two cases of the FOS have provided an insight into technologies that could be adapted for sensor-based material sorting. The general problem of extracting valuable materials from the commingled waste stream has a direct analogy to the security check for dangerous or forbidden objects, for example, in airports. Security scanners are at the cutting edge of technology, as they may use several kinds of radiation (X-ray, gamma and neutron) to classify materials in opaque containers [41]. With the recent trend for developing compact, portable, low-cost devices for threat detection (and related to that non-destructive testing) it becomes a fruitful source of inspiration for material recognition in recycling. For example, a technique that uses infrared stars of various wavelength to see through materials may become an affordable and safe replacement for X-ray scanners on sorting conveyors [42]. The technique is also remarkable for its use of mathematically-proven signal amplification method, which obviously cannot be found in physical effects databases.

Another FOS was run to address the existing gap between the ever-increasing resolution of sensors (such as hyperspectral cameras) and the dominant ejection method with an array of pneumatic valves, the so-called air jet blow bar. Although the nozzles can be placed as close as $3 \mathrm{~mm}$ from each other, their operation may lead to co-ejection of particles that belong to different fractions. Consequently, the waste is spread on the conveyor belt, so that it barely covers $5-10 \%$ of its area [43]. At the same time, air jets may divide the material flow into two, at the most three fractions, while sensors may classify several materials at once. From the viewpoint of energy consumption, the solution cannot be optimal either, as 
it is an inherent property of pneumatic circuitry to waste about $80 \%$ of energy [35]. The general problem statement, in this case, is how to deliver specific particles from a dense stream to the correct collection points. One of the ultimate leader in this respect is electrophotography that is used in laser printers. Indeed, a typical printer can deliver toner particles at the precision of several hundred dots per inch. The technology needs modification or even a completely different embodiment for adoption in the domain of recycling. Precisely activated electroadhesive devices should eventually outperform pneumatic ejectors in the waste sorting.

\section{Discussion}

The collected database of technologies has an advantage over paper reviews in that it is extendable with new information and can immediately show comparative infographics for the metrics chosen by the user (whose preferences may change over time). The tree-like storage of the data helps to cope with different level of details provided in publications, though some valuable information (such as price) remained in unstructured textual comments alongside with other categorical and numerical data. The developed software, however, lacks material modelling and simulation aids. The decision analysis is left outside and can be both algorithmic (e.g. Multi-Criteria Analysis) or merely judgmental. For example, in the case study [13] concerning the separation of plastics from construction and demolition waste the technologies were assessed as to which of them sort by the most divergent properties of the target materials and can sort them with associated impeding factors (dirt, moisture etc.). In the space of technological foresight, the conducted analysis leaned towards Monitoring and mapping class [9], as the study was conducted mainly through the literature search, enhanced by creativity approaches of TRIZ for ideation [45] and wider coverage of alternatives [10].

The effects-based approach takes the understanding of technologies and processes at another abstraction level that drives the search beyond the domain of the problem. A set of corresponding effects can be thought as a "genetic code" of the technology, which, in turn, allows to construct phylogenetic trees of technologies. Phylogenetic trees (see Figure 4) are built using dedicated algorithms from bioinformatics for comparison of genomes and they visualize the genetic relationship between species, as well as the process of evolutionary development. Differences in genomes help to set borders between species, just as they facilitate classification of technologies.

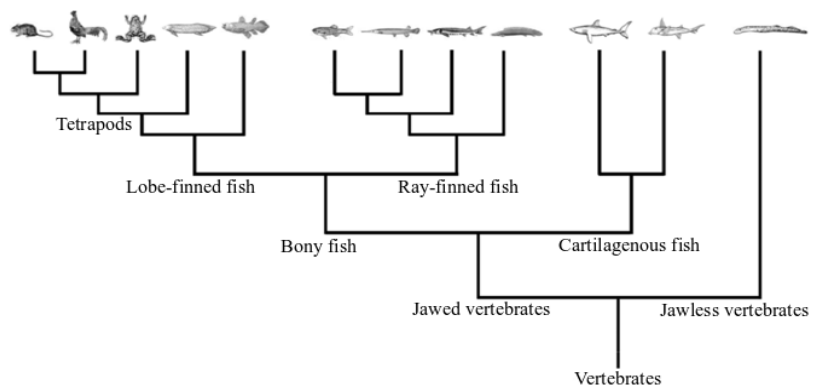

Figure 5. Example of a phylogenetic tree, adapted from [44].

The idea of technological "genes" has already appeared in [46], where the morphological analysis of textual description in patents provided "chromosomes" of technologies that were fed into a genetic algorithm to get more versions of a particular technology. The quality of a "chromosome" was set proportional to the number of patents reflecting its structure. If a technological "genotype" (a set of genes for a particular mechanism) was explicit enough to reflect quantitatively the actual topology and physics of operation it could be used for the evolutionary optimization algorithm to search for a completely new solution and test in a multiphysics simulation environment. Evolutionary computing has shown in some 
cases enough creativity to outsmart researchers that set seemingly unsolvable problems to their algorithms [47].

A plain set of effects is a vague representation of technology, but it can be firmly concluded that the effects databases need enhancement with similar building blocks from information technology, the algorithms. There can be a true synergy between sensing hardware and data processing software that will result in qualitatively new detection capabilities, as was demonstrated in [30]. Awareness of such possibilities can become a competitive advantage in innovation.

The FOS seems to fall off from the paradigm of the strong problem-solving analytical tools of TRIZ, like the substance-field analysis, resource analysis, inventive principles and many more. Its application for innovation in process engineering stalled since the 2000s [11]. However, it is a ready-to-use heuristic to make a well-guessed jump into another industrial field and escape from a local optimum of domainspecific technologies towards the best globally available technique.

\section{Concluding remarks}

The broad scope of the conducted technological survey impeded the use of many TRIZ tools aimed at defining inventive problems and resolving contradictions, however, even loose and fragmentary application of TRIZ principles promotes feasible search directions. New terms that are hardly associated with the domain under investigation lead to device concepts of considerable potential, as it was demonstrated in this work. The success of the conducted survey and ideation can only be proven by future implementation and testing of the concepts in practice, as the quantitative evaluation was hardly possible in such an early stage.

\section{Acknowledgements}

The authors thank Prof. Pavel Livotov for invaluable comments provided in preparation of the present paper, as well as anonymous reviewers for critical remarks.

\section{References}

1. Ragaert, K., Delva, L., Van Geem, K.: Mechanical and chemical recycling of solid plastic waste. Waste Management 69, 24-58 (2017).

2. Mellor, W., Wright, E., Clift, R., Azapagic, A., Stevens, G.: A mathematical model and decision-support framework for material recovery, recycling and cascaded use. Chemical Engineering Science, 57(22), pp. 4697-4713 (2002).

3. Van Schaik, A., Reuter, M. A.: Dynamic modelling of E-waste recycling system performance based on product design. Minerals Engineering, 23(3), pp. 192-210 (2010).

4. Ip, K., Testa, M., Raymond, A., Graves, S., Gutowski, T.: Performance evaluation of material separation in a material recovery facility using a network flow model. Resources, Conservation \& Recycling, 131, pp. 192205 (2018).

5. Gundupalli, S. P., Hait, S., Thakur, A.: A review on automated sorting of source-separated municipal solid waste for recycling. Waste Management, 60(C), pp. 56-74 (2017).

6. Singh, N., Hui, D., Singh, R., Ahuja, I.P.S., Feo, L., Fraternali, F.: Recycling of plastic sol-id waste: A state of art review and future applications. Composites Part B, 115(C), pp. 409-422 (2017).

7. Nabil, N.Z.G., Bülent, C., Hodgson, A.: Technology roadmapping for the next generation manufacturing enterprise., School of Mechanical, Materials and Manufacturing Engineering, University of Nottingham. Journal of Manufacturing Technology Management, 17(4), pp.404-416 (2006). 
8. Dully, S.: Internetbasiertes Technologie-Scouting für Recyclingtechnologien; Internet-based technology scouting for recycling technologies. Doctoral dissertation. Jost-Jetter Verlag, Heimsheim, Germany (2011).

9. Słupiński, M.: Technology Forecasting - State of the Art Update. FORMAT Consortium Deliverable (2013).

10. Porter, A.L.: Technology foresight: types and methods. International Journal of Foresight and Innovation Policy, 6(1-3), pp.36-45 (2010).

11. Livotov, P., Sekaran, A. P. C., Law, R., Reay, D., Sarsenova, A., Sayyareh, S.: Eco-innovation in process engineering: Contradictions, inventive principles and methods. Thermal Science and Engineering Progress, 9, pp. 52-65 (2019).

12. Litvin, S.: New TRIZ-based tool - function-oriented search (FOS). The TRIZ Journal, Au-gust (2005).

13. Ivanovskis, L.: Systematic selection of perspective solid waste mechanical separation technologies for material recovery. Master Thesis. LUT University (2019). Available: http://urn.fi/URN:NBN:fife201903148916, last accessed 2019/06/20.

14. Aulive Production Inspiration, http://www.productioninspiration.com, last accessed 2019/06/20.

15. Oxford Creativity TRIZ Effects Database, https://www.triz.co.uk/how/triz-effects-database, last accessed 2019/06/20.

16. Matmatch - Find Materials \& Suppliers, https://matmatch.com/, last accessed 2019/06/20.

17. Gent, M.R., Menéndez, M., Toraño, J., Torno, S.: Enhanced plastics recycling by cyclone media separation. In: ISWA APESB World Congress, Lisbon, Portugal (2009).

18. Andritz Homepage, https://www.andritz.com, last accessed 2019/02/13.

19. Carvalho, M.T., Agante, E., Durão, F.: Recovery of PET from packaging plastics mixtures by wet shaking table. Waste Management, 27(12), pp. 1747-1754 (2007).

20. Lupo, E., Moroni, M., La Marca, F., Fulco, S., Pinzi, V.: Investigation on an innovative technology for wet separation of plastic wastes. Waste management, 51, pp. 3-12 (2016).

21. Ito, M., Tsunekawa, M., Ishida, E., Kawai, K., Takahashi, T., Abe, N. and Hiroyoshi, N.: Reverse jig separation of shredded floating plastics - separation of polypropylene and high density polyethylene. International Journal of Mineral Processing, 97(1-4), pp. 96-99 (2010).

22. Kinoshita, T., Okamoto, K., Yamaguchi, K. and Akita, S.: Separation of plastic mixtures us-ing liquidfluidized bed technology. Chemosphere, 63(6), pp. 893-902 (2006).

23. Lee, M. J., Rahimifard, S. (2012). A novel separation process for recycling of post-consumer products. CIRP annals, 61(1), 35-38.

24. EP2679310. European Patent Office (2014).

25. Bakker, E. J., Rem, P. C., \& Fraunholcz, N.: Upgrading mixed polyolefin waste with magnetic density separation. Waste Management, 29(5), 1712-1717 (2009).

26. Wang, H., Mustaffar, A., Phan, A. N., Zivkovic, V., Reay, D., Law, R., Boodhoo, K.: A re-view of process intensification applied to solids handling. Chemical Engineering and Processing: Process Intensification, 118, pp. 78-107 (2017).

27. Lungu, M. and Neculae, A., 2018. Eddy current separation of small nonferrous particles using a complementary air-water method. Separation Science and Technology, 53(1), pp.126-135.

28. Dodbiba, G., Sadaki, J., Okaya, K., Shibayama, A., Fujita, T.: The use of air tabling and triboelectric separation for separating a mixture of three plastics. Minerals Engineering, 18(15), pp. 1350-1360 (2005).

29. Coanda, Inc. Coanda Process Screen, http://www.coanda.com/products/processscreen.htm, last accessed 2019/06/20.

30. Powder Systems Co. Small classifier, https://www.powder.co.jp/EN/products/01/, last accessed 2019/06/20.

31. Super, M.S., Enick, R.M., Beckman, E.J.: Density-based separation of thermoplastics found in the postconsumer waste stream. Resources, Conservation and Recycling, 9(1-2), pp. 75-88 (1993).

32. Catherall, A.T., Lopez-Alcaraz, P., Benedict, K.A., King, P.J., Eaves, L.: Cryogenically enhanced magnetoArchimedes levitation. New Journal of Physics, 7(1), art. no. 118 (2005).

33. Zhou, C., Pan, Y., Lu, M. and Yang, C.: Liberation characteristics after cryogenic modification and air table separation of discarded printed circuit boards. Journal of Hazardous Materials, 311, pp. 203-209 (2016).

34. Bjørk, R., Nielsen, K. K., Bahl, C. R., Smith, A., Wulff, A. C.: Comparing superconducting and permanent magnets for magnetic refrigeration. AIP Advances, 6(5), 056205 (2016). 
35. Máša, V., Kuba, P.: Efficient use of compressed air for dry ice blasting. Journal of Cleaner Production, 111, pp. 76-84 (2016).

36. ASTM D1525-00 Standard Test Method for Vicat Softening Temperature of Plastics. West Conshohocken: ASTM International, West Conshohocken. 8 p. (2000).

37. Bollegraaf Recycling Solutions: Paper Spike, http://www.fissoreagency.com/pages/wpcontent/uploads/2009/09/Paper-Spike.pdf, last accessed 2019/06/20.

38. EP1291092. European Patent Office (2003).

39. Tominaga, S. and Tanaka, N.: Refractive index estimation and color image rendering. Pattern Recognition Letters, 24(11), pp. 1703-1713 (2003).

40. Saman, G. and Hancock, E.: Refractive index estimation using photometric stereo. In 2011 18th IEEE International Conference on Image Processing, pp. 1925-1928 (2011).

41. Sowerby, B. and Tickner, J.: Scanner for the detection of contraband in air cargo containers. The Physicist"Australian Institute of Physics, 42(10) (2005).

42. Senni, L., Burrascano, P. and Ricci, M.: Multispectral laser imaging for advanced food analysis. Infrared Physics \& Technology, 77, pp. 179-192 (2016).

43. Yu, H.: Metal Recovery via Automated Sortation. Doctoral dissertation. Worcester Polytechnic Institute (2014). Available: https://web.wpi.edu/Pubs/ETD/Available/etd-041714-211407/unrestricted/HaoYu.pdf, last accessed 2019/06/20.

44. Yamamoto, K., Bloch, S., Vernier, P.: New perspective on the regionalization of the anterior forebrain in Osteichthyes. Development, growth \& differentiation 59(4), pp. 175-187 (2017).

45. Ilevbare, I., Phaal, R., Probert, D., Padilla, A.T.: Integration of TRIZ and roadmapping for innovation, strategy, and problem solving. Centre for Technology Management, University of Cambridge, UK, and Dux Diligens, Mexico (2011).

46. Yoon, B.G., Jin S.Y.: Applications of Genetic Algorithm and Text Mining on Technology Innovation. Applied Mechanics and Materials, vol. 145, pp. 287-291 (2012).

47. Lehman, J., Clune, J., Misevic, D., Adami, C., Altenberg, L., Beaulieu, J., Bentley, P.J., Bernard, S., Beslon, G., Bryson, D.M., Chrabaszcz, P.: The surprising creativity of digital evolution: A collection of anecdotes from the evolutionary computation and artificial life re-search communities. arXiv preprint arXiv:1803.03453 (2018). 